\title{
Ethisierung und die Marginalisierung der Ethik
}

\author{
Zur Mikropolitik des Wissens in Ethikräten
}

\section{Von Alexander Bogner}

Zusammenfassung: In diesem Beitrag wird das organisationssoziologische Konzept der Mikropolitik für die Analyse von Ethikexpertise verwendet. Aus dieser Perspektive wird deutlich, dass im Rahmen interdisziplinärer Expertiseproduktion immer auch über die Geltungsansprüche disziplinären Sonderwissens verhandelt wird. In dieser Mikropolitik des Wissens entscheidet sich, wer erfolgreich Expertenstatus behaupten kann. Dies wird am Beispiel nationaler Ethikräte gezeigt. Die ethische Rahmung dieser Expertise führt nun, so die zentrale These, nicht zu einer Privilegierung sondern - im Gegenteil - zu einer Marginalisierung ethischen Wissens. Konstatiert wird damit eine gegenläufige Entwicklung: Während sich auf der Makroebene ein hegemonialer Trend zur Ethisierung wissenschaftlich-technischer Phänomene beobachten lässt, zeigt sich auf der operativen Mikroebene eine relative Marginalisierung der Fachethik.

Der folgende Beitrag hat zum Ziel, in theoretischer wie empirischer Hinsicht zu einer Soziologie der Ethikexpertise beizutragen. ${ }^{1}$ In theoretischer Hinsicht wird der Versuch unternommen, das organisationssoziologische Konzept der Mikropolitik für wissenschaftssoziologische Interessen nutzbar zu machen. Dies eröffnet die Möglichkeit, die Aushandlung von Expertise in interdisziplinären Expertengremien als Mikropolitik des Wissens zu analysieren. Es wird gezeigt, dass in interdisziplinären Expertengremien immer auch über die Geltungsansprüche disziplinären Sonderwissens verhandelt wird. Diese Auseinandersetzungen um Wissensansprüche werden in wechselnden Experten-Laien-Konstellationen ausgetragen schließlich ist jeder Fachexperte in der Nachbar(sub)disziplin ein Laie. ${ }^{2}$ Mit dem Bezug auf Mikropolitik wird Interdisziplinarität also aus einer dezidiert machtanalytischen Perspektive analysiert. Mikropolitik des Wissens erscheint als ein notwendiger Bestandteil interdisziplinären Arbeitens.

In dieser Mikropolitik entscheidet sich: Welches Wissen zählt als Wissen? Was gilt als (gutes) Argument, was als relevante Kritik? Wem gelingt es, seine Wirklichkeitsinterpretation handlungs- und entscheidungsrelevant werden zu lassen? Wer kann erfolgreich Expertenstatus behaupten? Und, Stichwort „wechselnde Experten-Laien-Konstellationen“: Von wem und auf welcher Ebene sind Wissens- und Deutungsansprüche bestreitbar? Das Erkenntnisinteresse richtet sich also darauf, welche „Hierarchie von Wissensformen“ (Bora 2009) sich im Rahmen interdisziplinärer Expertise etabliert.

Dass es sich beim empirischen Fallbeispiel um Nationale Ethikräte handelt, macht den besonderen Reiz dieser Studie und ihre Relevanz für eine Soziologie der Ethik aus. Denn damit wird die Frage virulent, welche Folgen diese Kommissionsethik für die professionelle Ethik hat. Wird die Ethik zur „Dachwissenschaft“, die alle anderen Disziplinen zu Hilfswissenschaften degradiert? Im Folgenden wird argumentiert, dass genau dies nicht der Fall ist. Zwar beobachten wir einerseits eine diskursive Entgrenzung der Ethik, die unter anderem

1 Intellektuelle und moralische Unterstützung verdanke ich einem anonymen Gutachten sowie Wolfgang Menz.

2 Die verweist im Übrigen auf den implizit transdisziplinären Charakter interdisziplinären Arbeitens, auf den - wenn auch mit anderer Akzentsetzung - schon Jürgen Mittelstraß (1987) hingewiesen hat: Wenn Interdisziplinarität wirklich als Dialog über Fächergrenzen hinweg geführt wird, sitzen die Laien immer schon mit am Beratungstisch der Experten, und zwar im konkreten Fall - anders als im Partizipationskonzept von Nowotny et al. (2001) - in Form renommierter Fachexperten.

Soziale Welt 60 (2009), S. $119-137$ 
die Forschungs- und Technikregulierung als Aufgabe ethischer Reflexion kennzeichnet (Ethisierung). Andererseits lässt sich jedoch feststellen, dass die Organisation der ethischen Reflexion in Form interdisziplinärer Expertengremien zu einer Infragestellung des ethischen Definitionsmonopols führt. Konstatiert wird also eine gegenläufige Entwicklung: Während sich auf der Makroebene ein hegemonialer Trend zur Ethisierung wissenschaftlich-technischer Phänomene beobachten lässt, zeigt sich auf der operativen Mikroebene eine relative Marginalisierung der Fachethik.

Diese These wird in fünf Schritten entwickelt. Zunächst wird der Begriff der Ethisierung präzisiert. Ethisierung wird als eine bestimmte Thematisierungsperspektive von wissenschaftlich-technischen Phänomenen verstanden, die sich gerade in Abgrenzung zum Risikodiskurs deutlich profilieren lässt (1). Anschließend wird mit Rekurs auf die Organisationstheorie der Begriff der Mikropolitik präzisiert. Dies schafft die Grundlage, um die Aushandlungen in den Ethikgremien als Kampf um die Anerkennung von Wissen analysieren zu können (2). Danach wird eine Systematisierung unterschiedlicher Geltungskriterien vorgestellt, die für die Bestreitung von Wissensansprüchen innerhalb der Kommissionen eine Rolle spielen. Dahinter steckt die Vorstellung, dass sich die Geltung von Wissen aus einer Kombination unterschiedlicher Geltungskriterien ergibt (3). Im Mittelpunkt der empirischen Analyse steht die Frage, welche Folgen ethische Deliberation für die interne Wissensordnung hat. Dabei richtet sich der Fokus auf die professionelle Ethik. Es wird gezeigt, dass sich in nationalen Ethikräten eine relative Marginalisierung der Fachethik beobachten lässt (4). Dies wird gerade im Vergleich mit juristischer Expertise deutlich. Die Dominanz der Jurisprudenz äußert sich darin, dass sie auch als Kommissionsrecht sachlich und sozial unangefochten bleibt (5). Abschließend werden die empirischen Befunde resümiert und im Hinblick auf die Entwicklung einer - über die Soziologie der Ethikexpertise hinaus reichende - Soziologie der Ethik gewichtet (6).

\section{Ethisierung: Die Ethik als Rahmen}

Die Mikropolitik des Wissens wird im Folgenden am Beispiel von Politikberatungsgremien im Bereich der Bioethik dargestellt. In vielen westlichen Demokratien wurden in den letzten Jahren nationale Bioethikkommissionen gegründet (Fuchs 2005; Galloux et al. 2002). In Deutschland hatte Kanzler Gerhard Schröder im Sommer 2001 einen Nationalen Ethikrat einberufen. Seit Ende 2007 heißt er Deutscher Ethikrat und operiert - anders als sein Vorgänger - auf Basis einer gesetzlichen Grundlage. In Österreich wurde im selben Jahr eine Bioethikkommission beim Bundeskanzleramt eingerichtet; und in der Schweiz hatte der Bundesrat ebenfalls 2001 eine Nationale Ethikkommission im Bereich Humanmedizin konstituiert, jedoch nach einer wesentlich längeren Vorlaufphase und ausführlicher parlamentarischer Beratung.

Diese nationalen Ethikräte, die über das Für und Wider von Stammzellforschung, Sterbehilfe oder Gendiagnostik beraten, sind interdisziplinär besetzt - auch wenn die disziplinäre Zuordnung im Einzelfall oft schwierig sein mag und die Experten außerdem in der Praxis nicht (immer) unbedingt als Disziplinvertreter auftreten müssen. ${ }^{3}$ Die Kommissionsförmigkeit der Ethikberatung bedeutet in jedem Fall, dass Ethikexpertise ein Produkt ist, das zwischen Vertretern verschiedener Disziplinen und Weltanschauungen ausgehandelt werden

3 In den Einrichtungsverordnungen der Ethikräte heißt es dazu, dass Expertise aus verschiedenen Wissenschaftsgebieten vertreten sein soll, wobei Naturwissenschaften und Medizin meist an erster Stelle genannt werden - vor den Juristen, Philosophen und Theologen. Nur in der Schweizer Verordnung steht die Ethik an erster Stelle. In den USA oder Österreich dagegen wird die Ethik gar nicht als eigenständige Disziplin aufgeführt. Im Anschluss an die Auflistung der Disziplinen wird meist darauf hingewiesen, dass ein plurales Meinungsspektrum vertreten sein soll. 
muss. Ethikexpertise hat nicht die Form des Einzelgutachtens oder redaktionell synthetisierter Einzelgutachten, etwa im Stil der Sachstandsberichte aus der Technikfolgenabschätzung. $\mathrm{Zu}$ den großen bioethischen Streitfragen publizieren nationale Ethikräte meist umfangreiche Stellungnahmen inklusive politischer Handlungsempfehlungen, deren Heterogenität den oft unüberwindlichen Expertendissens zum Ausdruck bringt. Dieser Ethikexpertise kommt in der Regel erhebliche mediale Aufmerksamkeit und auch eine gewisse Relevanz für politische Entscheidungsprozesse zu (Bogner et al. 2008).

Der Trend zur Institutionalisierung interdisziplinärer Ethikberatung lässt sich mit der verschiedentlich konstatierten Ethisierung von Wissenschafts- und Technikkontroversen in Verbindung bringen (Lindsey et al. 2001; Maasen 2002; Braun et al. 2008). Ethisierung heißt: Problematisierung von Forschung und Technologien sowie Prozessierung und Regulierung dieser Konflikte in ethischen Kategorien. ${ }^{4}$ Mit anderen Worten: Die Ethik stellt den maßgeblichen Rahmen (oder Frame) für diese Auseinandersetzungen dar, v.a. im Bereich der Biomedizin. Frames sind dabei als machtvolle Organisationsprinzipien von Interaktionen zu verstehen, denen die Funktion zukommt, eine gemeinsame diskursive Basis für die Austragung von Konflikten herzustellen. ${ }^{5}$ Schließlich bedürfen Konflikte, wie schon Georg Simmel (1958) betont hat, gemeinsam geteilter Relevanzen, um überhaupt ausgetragen werden zu können. Ohne gemeinsam geteilten Rahmen gibt es nicht Dissens, sondern Indifferenz.

Dies sensibilisiert für die latenten Machtwirkungen von Frames: Zum einen steuern und strukturieren Frames unsere Seh-, Denk- und Handlungsgewohnheiten; zum anderen determinieren Frames Kontroversen insofern, als die Kontrahenten sich konstruktiv auf etablierte Frames beziehen müssen. Für Biomedizin-Kontroversen heißt das: Politiker und Forscher können ihr Plädoyer für die Stammzellforschung nicht einfach nur ökonomisch begründen („Standortsicherung“); sie müssen es immer auch ethisch reformulieren. Zu diesem Zweck ist der Rekurs auf eine „Ethik des Heilens“ sehr populär geworden (Rubin 2008).

Wissenschafts- und Technikkontroversen müssen nicht als ethisches Problem ausgetragen werden. Beispiel grüne Gentechnik: In der Kontroverse um die Freisetzung gentechnisch veränderter Pflanzen wird nicht über das moralisch Gebotene gestritten, sondern um das richtige Wissen. Im Mittelpunkt steht die Frage: Wie hoch ist das Risiko eines Natureingriffs? Welche ökologischen und gesundheitlichen Gefahren resultieren aus dem Versuch, Pflanzen mit Hilfe der Gentechnik resistenter gegen Schädlinge zu machen? Die grüne Gentechnik wird also in erster Linie als ein Risiko-Problem verhandelt, nicht als Ethik-Problem. Es wird um Sicherheitsbehauptungen und Risikoeinschätzungen gestritten, nicht um den Wert des Lebens.

4 Ethisierung wird hier nur im Hinblick auf Wissenschaft und Technik verhandelt. Sie lässt sich als Teil eines Ethik-Booms begreifen, der auch von distanzierten Beobachtern diagnostiziert wird (Luhmann 1997: 156), und darum viel weiter fassen: etwa als Modus individueller Kaufentscheidungen und damit als Teil der Ökonomie (Moorstedt 2007; Stehr 2006); als Bezugsrahmen politischer Konfliktaufbereitung (Mouffe 2007), man denke an „Schurkenstaaten“ oder die „Achse des Bösen“; oder auch als politische Technologie der Selbststeuerung in Form diverser Beteiligungs- und Vorsorgeimperative (Maasen / Kaiser 2007; Sutter 2005; Rose 2001).

5 Frames beinhalten ,underlying structures of belief, perception, and appreciation“ (Schön / Rein 1995: 23), die in politischen Auseinandersetzungen relevant werden. Allerdings werden in der PolicyForschung Frames in der Regel zugleich mit spezifischen inhaltlichen Positionierungen in Verbindung gebracht (vgl. Entman 1993). Politische und gesellschaftliche Kontroversen werden somit primär als Konfrontation unterschiedlicher Rahmungen aufgefasst. Demgegenüber zielt der hier verwendete Frame-Begriff darauf herauszufinden, auf welche gemeinsamen Begriffe, normativen Annahmen und Diskurse sich die Kontrahenten in ihren Auseinandersetzungen beziehen (und beziehen müssen, um anschlussfähig zu werden). Ethik wird damit als ein „Basis-Frame“ im Sinne Dahindens aufgefasst (Dahinden 2006: 105ff, 210ff). 
Natürlich: Hinter der Kritik an der grünen Gentechnik stehen nicht nur alternative RisikoKalkulationen, sondern auch ganz andere Werthaltungen, etwa ein alternatives Naturverständnis (Gill 2003). Doch der dominante Expertendiskurs wurde und wird weitgehend als Wissenskonflikt gerahmt. Ein gutes Beispiel dafür bietet das WZB-Verfahren zur Herbizidresistenz (van den Daele et al. 1996). Den Kern der dortigen Auseinandersetzungen von Experten und Gegen-Experten bildeten konfligierende Wahrheitsansprüche, nicht Wertkonflikte. ${ }^{6}$

Im Prinzip lässt sich die spezifische Rahmung einer Kontroverse also mit der Privilegierung eines bestimmten Wissens in Verbindung bringen. Im Fall der Risikokontroversen etwa spielt naturwissenschaftliches Expertenwissen, nicht zuletzt aufgrund seines Eindeutigkeitsund Objektivitätsversprechens, zweifellos eine besondere Rolle. Ob in bioethischen Wertkonflikten fachethisches Expertenwissen eine ähnlich tragende Bedeutung erhält, bleibt zu prüfen. In jedem Fall sensibilisiert das Frame-Konzept für das potenzielle Spannungsverhältnis zwischen Diskurs und Wissen. So erlaubt es z.B. im Hinblick auf Ethikexpertise zu fragen, ob der für die spezifische Institutionalisierungsform von Expertise maßgebliche Diskurs die Ethik - auf Ebene der Aushandlungsprozesse einem bestimmten Wissen besondere Geltung verschafft. Die für die empirische Analyse konstitutive Frage nach der Relevanz ethischen Fachwissens steht damit nicht außerhalb oder quer zu dem hier präsentierten FrameKonzept, sie ergibt sich vielmehr daraus.

\section{Mikropolitik des Wissens}

Um Nutzen und Grenzen des Mikropolitik-Ansatzes für wissenschaftssoziologische Analysen diskutieren zu können, bedarf es einer kurzen Einordnung dieses Konzepts.

Mikropolitik und mikropolitisches Handeln sind im Zusammenhang mit Organisationen (und hier vor allem mit Unternehmen) zum Thema geworden. ${ }^{7}$ Auch wenn (erwartungsgemäß) keine einheitliche oder allgemein überzeugende Definition von „Mikropolitik“ vorliegt (Neuberger 2006), so bezeichnet der Begriff innerhalb der Organisationssoziologie doch eine ganz bestimmte Problematisierungsperspektive. Der Fokus auf Mikropolitik verdankt sich einer Kritik am Rationalmodell der Organisation (Elšik 1997). In diesem Modell erscheinen Organisationen als durch stabile Strukturen bestimmte und nach Maßgabe der Zweckrationalität funktionierende Maschinen. Aus mikropolitischer Perspektive werden Organisationen dagegen als Handlungsgeflecht von verschiedenen Akteuren mit heterogenen Zielen und Interessen verstanden. Dementsprechend erscheint die Organisation nicht länger als Maschine, sondern als Spielfeld (Crozier / Friedberg 1993) oder Arena (Türk 1993). Der Fokus richtet sich auf politische Prozesse, die durch Handlungsspielräume für Individual- oder Kollektivakteure ermöglicht und zur Verfolgung eigener Interessen genutzt werden. Es sind demnach nicht Ressourcenknappheit oder bestimmte Persönlichkeitsmerkmale (vgl. Bosetzky 1977), die zu Mikropolitik führen. Mikropolitik hat vielmehr mit grundsätzlichen Bedingungen organisatorischen Handelns zu tun, nämlich einem prinzipiell mangelhaften Regeldeterminismus und (zumindest minimaler) Handlungsautonomie. Eine solche Perspektive ist erkennbar gegen einen Strukturdeterminismus marxistischer oder funktionalistischer Prägung gerichtet.

Mit der Rehabilitierung von Subjektivität, Informalität und Indeterminiertheit in der Organisationsanalyse ergeben sich Anschlussmöglichkeiten an neuere, poststrukturalistisch ge-

6 „Der Streit der Konfliktparteien betraf nicht die normativen Prinzipien - über Selbstverständlichkeiten streitet man nicht. Er betraf die empirischen Voraussetzungen des moralischen Urteils: Sind schädliche Folgen gentechnisch veränderter Pflanzen tatsächlich zu erwarten?“ (van den Daele 2001: 10).

7 In der Politikwissenschaft wurde ein mikropolitischer Ansatz von Nullmeier et al. (2003) auf dem Feld der Policy-Analyse eingeführt, und zwar unter dem Begriff „Mikro-Policy-Analyse“. Dabei geht es um die Erforschung der Mikrostrukturen und -prozesse innerhalb eines Politikfelds. 
prägte Analysen (Kneer 2008; Knights 1997). Eine grundlegende Voraussetzung dafür ist die Anerkennung der Kontingenz, d.h. die Annahme, dass Handeln im Medium von Strukturen stattfindet, ohne jedoch hierauf rückführbar zu sein (Friedberg 1992: 39). Die Betonung der Kontingenz unterstreicht den zentralen Stellenwert, der der Macht in diesen Analysen zukommt. Macht, so halten Crozier und Friedberg in ihrer einflussreichen Studie „Über Macht und Organisation“ fest, ist die „unausweichliche Dimension jeder sozialen Beziehung“ (1993: 17). Macht gilt demzufolge nicht als Defekt oder Negativum (Repression, Gewalt), sondern als normal und allgegenwärtig. Soziale Beziehungen sind immer Machtbeziehungen, sofern man die Akteure als relativ autonom und nicht als reine Marionetten begreift. Der Mikropolitik-Ansatz impliziert außerdem - wiederum in Abgrenzung zum Marxismus -, dass Machtbeziehungen nicht unilinear von oben nach unten verlaufen, sondern sich dezentral und lokal in einer Vielfalt von Kräfteverhältnissen konstituieren (Küpper / Ortmann 1986). Damit verbindet sich das spezifische Interesse an den jeweils besonderen, lokalen Konstitutionsbedingungen, den Praktiken und Techniken von Macht. Macht erscheint auf diese Weise nicht als Besitztum, das sich im Alltag manifestiert oder repräsentiert, sondern als etwas, das ausgeübt wird und sich in der Beziehung zwischen Akteuren herstellt. All dies verweist auf eine - nur selten systematisch reflektierte (Ortmann 1992) - Macht-Konzeption, die eine große Nähe zu Foucault aufweist (vgl. Foucault 1983: 113ff; 1994), obwohl der meist nur beiläufig und in der strategischen Organisationsanalyse gar nicht erwähnt wird.

In der Organisationssoziologie ist mittlerweile auch die Mikropolitik des Wissens explizit zum Gegenstand theoretischer und empirischer Analysen geworden. Die Betonung liegt hier auf explizit, denn tatsächlich ist ja die Berücksichtigung mikropolitischer Aspekte in der Elaborierung von Wissen und wissenschaftlicher Wahrheit in einer konstruktivistisch ausgerichteten Wissenschaftssoziologie gute Tradition. Dass wissenschaftliche Tatsachen nicht Repräsentationen sind, sondern in einem kollektiven Prozess geformt werden müssen, hat schon Ludwik Fleck (1994: 117 f) betont. Das Erkenntnisinteresse späterer Analysen von Interaktionsprozessen in Labors ließe sich immerhin zu einem Teil unter mikropolitischer Perspektive reformulieren (Knorr-Cetina 1984). Das heißtt, hier existiert eine Forschungstradition, die für das Konzept einer Mikropolitik des Wissens erhebliche Bedeutung hat, aufgrund ihrer Fokussierung auf Forschung aber nicht in den Blick der „Mikropolitologie“ gerät.

In organisationssoziologischen Zugängen ist das Expertenwissen hinsichtlich seiner Anschlussfähigkeit und Relevanz für organisationale Entscheidungsprozesse von Interesse. Es geht also vordringlich darum, das Verhältnis zwischen Wissen und Entscheiden besser zu verstehen. Daraus ergeben sich zwei unterschiedliche, aber einander nicht ausschließende Analyseperspektiven. Zum einen lässt sich fragen, welche Rolle professionelle Expertise im Kontext mikropolitischer Taktiken und Strategien hat. Damit gerät Expertise als Mittel und Ressource von Mikropolitik in den Blick. Die Politik mit Expertise wird zu einem weiteren der vielen Tricks im täglichen Machtspiel (Weißbach 2001). Zum anderen - aus einer stärker wissenssoziologisch angeleiteten Perspektive - kann Mikropolitik als konstitutiv für die gemeinsame Elaborierung und damit für die Anerkennung von Expertenwissen verstanden werden, so etwa bei Lazega (1992), der in seiner Analyse auf Berger und Luckmann rekurriert. Er geht davon aus, dass das, was als Wissen zählt, vom Prozess einer interaktiven Elaborierung von Information abhängt. In diesem Prozess werden Wissensansprüche (,knowledge claims") erhoben, je nach Rolle, Autorität und Gruppenzugehörigkeit in unterschiedlicher Form; außerdem werden Kriterien ausgehandelt, nach denen die Anschlussfähigkeit des Expertenwissens beurteilt wird (,appropriateness judgements“). Damit wird deutlich, dass die interaktive Aushandlung von Wissensansprüchen einen deutlichen Einfluss darauf hat, was am Ende als informierte Entscheidung gelten wird. 
Für die Wissenschaftssoziologie sind diese Analysen zur Mikropolitik des Wissens instruktiv, weil sie - vor dem Hintergrund einer wachsenden Bedeutung von Expertise für betriebliche Modernisierungsprozesse - die Wirkmächtigkeit von Expertenwissen als Ergebnis von Aushandlungsprozessen interpretieren. Jedoch bekommt die Mikropolitik des Wissens im Kontext einer Mikrosoziologie von Ethikexpertise eine etwas anders gefärbte Semantik. Die Mitglieder in Organisationen vom Typ Ethikrat sind in der Regel Experten. Das heißt zum einen, dass Wissensansprüche kaum auf Status und formale Autorität setzen können. ${ }^{8}$ Vor allem aber kann das Expertenwissen nicht als homogene Entität, etwa in Abgrenzung zu nichtprofessionellen Wissensformen, konzeptualisiert werden. Eine mikropolitische Analyse der interdisziplinären Erstellung von Expertise interessiert sich gerade für Differenzierungs- und Hierarchisierungsprozesse innerhalb des Expertenwissens.

Eher beiläufig wurde bisher im Rekurs auf Mikropolitik erwähnt, dass wir es im Fall der nationalen Ethikräte mit Organisationen zu tun haben. Genau dies dürfte allerdings erhebliche Auswirkungen auf die internen Aushandlungen von Wissensansprüchen haben. Denn aus differenzierungstheoretischer Perspektive stellen Ethikräte multi-referenzielle Organisationen dar, die die Relevanz- und Gültigkeitskriterien unterschiedlicher Funktionssysteme integrieren (Jung 2009: 75ff); sie sind gewissermaßen immer schon „boundary organizations“ (Guston 2001), weil sie divergierende Handlungsorientierungen und Ansprüche einander vermitteln müssen. Ethikräte explizit als Organisationen zu kennzeichnen erfordert daher in der empirischen Analyse, die Hierarchisierung von Wissensformen auch auf den Aspekt der Multireferenzialität zu beziehen, und das heißt im konkreten Fall: die Notwendigkeit der Anschlussfähigkeit ethischer Deliberation für Politik und Wissenschaft zu berücksichtigen.

\section{Kriterien der Geltung von Wissen}

Die mikropolitische Perspektive gilt es nun im Hinblick auf das konkrete Fallbeispiel stärker zu operationalisieren. Eine der relevantesten Fragen lautet: Auf welcher Ebene können Wissensbehauptungen und Deutungsansprüche in einem interdisziplinären Gremium bestritten werden? Wie und von wem wird Wissen in Frage gestellt? Und wird es das denn überhaupt?

Es wäre ja auf den ersten Blick nicht völlig unplausibel davon auszugehen, dass mit dem Eintritt in eine Kommission Geltungsfragen verblassen. Man könnte etwa annehmen, dass sämtliche im Gremium vertretene Expertise automatisch als gültig und relevant erscheint. Die Frage nach der Bestreitungsmöglichkeit von Wissensansprüchen wäre dann obsolet. Die bloße Tatsache der Mitgliedschaft hätte diese Frage bereits entschieden. Eine solche Annahme ist jedoch im konkreten Fall nicht nur empirisch leicht widerlegbar. Sie ist auch theoretisch unbefriedigend, weil sie eine Harmonie in interdisziplinären Gremien unterstellt, die für die Analyse von Macht und Konflikt keinen Raum lässt.

Im Folgenden wird eine Systematisierung unterschiedlicher Geltungskriterien vorgestellt, die für die Bestreitung von Wissensansprüchen innerhalb der Kommissionen eine Rolle spielen. Zwecks einer ersten Orientierung mag es sich anbieten, in loser Anlehnung an Habermas eine dreidimensionale Unterscheidung von Geltungsansprüchen vorzunehmen. Da es sich in unserem Fall jedoch nicht um unterschiedliche „Welten“ handelt, auf die sich die Sprechakte beziehen, sind allerdings Modifikationen erforderlich. Für diese Modifikationen kann nicht allein auf Literatur zurückgegriffen werden. Die klassischen Texte zur Qualitätssicherung wissenschaftlicher Expertise (z.B. Clarke / Majone 1985; Jasanoff 1985) sind nur

8 Was nicht heißt, dass interdisziplinäre Gremien durch eine Gleichverteilung der Macht charakterisiert wären. Ohne jede Asymmetrie in den sozialen Beziehungen gäbe es gar keinen Angriffspunkt für Mikropolitik. Tatsächlich sind die Aushandlungsprozesse durch spezifische Erwartungsstrukturen geprägt (z.B. Status der Disziplin, Problem-Framing), die zur Kontingenzreduktion beitragen. 
bedingt hilfreich, weil sie auf die Sicherung politischer oder gesellschaftlicher Legitimität abheben (als Überblick vgl. Lentsch 2008; Weingart et al. 2007: 299ff). Und übergreifende Systematiken gremieninterner Geltungskriterien sind angesichts der Vielfalt von Expertise schwer vorstellbar. Daher ist die folgende Systematisierung gleichzeitig eine fallspezifische Rekonstruktionsleistung. Sie resultiert nicht zuletzt aus der empirischen Analyse der Praktiken selbst. Dabei ist diese Systematisierung von der grundlegenden Idee getragen, dass sich die Geltung von Wissen aus der Kombination einzelner Geltungsdimensionen ergibt: 1) Wahrheit, 2) Robustheit und 3) Relevanz.

1) Wahrheit bezeichnet die Ebene epistemischer Legitimität. Die Prüffrage lautet, ob ein Wissensanspruch den erkenntnistheoretisch abgestützten Kriterien, die von einer bestimmten peer group getragen werden, genügt. Wahrheitsprüfung heißt also: Wissensbehauptungen werden nach Maßgabe disziplinärer Qualitätsstandards beurteilt und können auf dieser Ebene auch sehr wirkungsvoll kritisiert werden. Diese Variante der Geltungsbestreitung wird die Auseinandersetzung in der Regel auf die Disziplinenvertreter einschränken. Zwischen diesen allerdings findet sie auch statt, denn selbstverständlich gibt es - oft schon über Grundtatbestände - erheblichen Dissens innerhalb einer (Sub-)Disziplin. Wer nicht mit den Wissenschaftlichkeitsstandards des betreffenden Fachs vertraut ist bzw. zur peer group gehört, kann nicht wirkungsvoll in diese Auseinandersetzung intervenieren - er muss vertrauen. Voraussetzung der Wahrheitsprüfung ist also Fachexpertise. Wahrheit - das ist die Geltungsprüfung durch den Wissenschaftler als Fachexperten.

2) Robustheit bezeichnet die Ebene sozial-moralischer Legitimität, d.h. den Aspekt der Bewährung von Wissen in einem weiteren Kontext (,viability“ im Sinne von Glasersfeld [1997: 49ff]). In unserem Zusammenhang ist dabei nicht der Anwendungsbezug des Wissens von Bedeutung; die Frage ist nicht, ob das Wissen in der „Realität“ funktioniert. Unser Thema ist die Wissenspolitik innerhalb von Expertengremien, nicht die gesellschaftliche Akzeptanz von Forschung. In diesem Sinne soll mit dem Begriff der Robustheit dafür sensibilisiert werden, dass wissenschaftliches Wissen unter bestimmten Umständen für Laienkritik anfällig werden kann. Diese Kritik basiert nicht auf einer ebenbürtigen Fachexpertise, sondern auf dem Wissen um maßgebliche Kontroversen im betreffenden Fachbereich, alternative Studien, Gegenpositionen usw. Wissensansprüche können damit nicht widerlegt, wohl aber mehr oder weniger wirkungsvoll in Frage gestellt werden. Dafür ist allerdings Kenntnis der jeweiligen Begrifflichkeiten, Methoden und Forschungslogik wichtig - man muss ungefähr wissen, wie das Fach funktioniert. Es bedarf also nicht Fachexpertise auf Basis von disziplinärem Sonderwissen, sondern dessen, was Collins und Evans (2007) als ,interactional expertise" bezeichnen: sich in einem Forschungsfeld sicher bewegen zu können, ohne die Mittel zur potenziellen Teilhabe an der Wissensproduktion zu haben. Robustheit - das ist die Geltungsprüfung durch den Wissenschaftler als (wissenschaftskritischen) Laien.

3) Relevanz bezeichnet die Ebene einer praktisch-diskursiven Legitimität. Es wird thematisiert, welche Bedeutung dem Wissen - ganz ungeachtet seiner Wahrheit und Robustheit für die Verhandlung der konkreten Problemstellung zukommt. Relevanz bezeichnet jene Dimension der Geltung, in der diskursive Zwänge eine Rolle spielen, die sich aus den Strukturbedingungen der Interaktion ergeben, wie z.B. der Funktion des Gremiums, dem spezifischen Status der Disziplin oder eben dem Problem-Framing. Das heißt nicht, dass die konkreten Interaktionen für den Relevanzstatus irrelevant würden, im Gegenteil. Relevanzansprüche können immer auch praktisch unterlaufen werden, indem beispielsweise die etablierte Problematisierungsperspektive im Gremium kritisiert und reformuliert wird. Hinsichtlich der Relevanz operiert der Experte weder als Wissenschaftler im Dienste der Wahrheit noch als Laie im Sinne der Glaubwürdigkeit. Wissensansprüche lassen sich auf Ebene der Relevanz nur wirksam problematisieren, indem man sich kritisch auf den politischen Kontext, 
die konkrete Aufgabenstellung oder auf den hegemonialen Diskurs bezieht (in unserem Fall: die Ethik). Relevanz - das ist die Geltungsprüfung durch den Wissenschaftler als Diskurspolitiker.

Natürlich ist diese Systematik als idealtypische Konstruktion zu heuristischen Zwecken zu verstehen. In der Praxis sind Unschärfen und Überlappungen zwischen den Typen erwartbar. Die Systematik verdeutlicht aber, dass die Geltung des Wissens in Expertenkommissionen als komplexe Konfiguration zu verstehen ist.

\section{Die Marginalisierung der Ethik}

Die eingangs formulierte These, wonach die Ethisierung von Wissenschafts- und Technikkontroversen eine relative Marginalisierung der Fachethik auf der Mikroebene bedeutet, wird im Folgenden anhand einer Mikroskopie interdisziplinärer Ethikexpertise entwickelt. Diese Mikroskopie führt die Erstellung von Ethikexpertise als Prozess der Aushandlung von Geltungsansprüchen vor. Dabei können nicht alle Disziplinen und Wissensformen gleichermaßen berücksichtigt werden. Im Folgenden wird insbesondere auf die Rolle fachethischer und juristischer Expertise fokussiert, weil sie in exemplarischer Weise kontrastieren. Außerdem vermitteln sie kontraintuitive Einsichten in die Logiken der Aushandlungsprozesse.

Marginalisierung der Ethik heißt in diesem Zusammenhang: Das ethische Sonderwissen ist nicht in der Lage, einen spezifischen Expertenstatus der Ethiker zu begründen - obwohl es um ethische Fragen geht. Diese Marginalisierung resultiert nicht aus der Geringschätzung der konkreten Ethik-Repräsentanten in den Gremien oder der Ethik als Profession; sie verdankt sich auch nicht einer fundamentalen Kritik der Ethik als Rahmen (dass man es mit substanziell ethischen Fragen zu tun habe, wird von niemandem bestritten). Es wird vielmehr gezeigt, dass die Marginalisierung mit konkreten Erwartungen, Strategien und Handlungszwängen zu tun hat, die aus der politischen Organisation von Ethikexpertise als Aushandlungsprozess unter Anwesenden resultieren.

Die Analyse stützt sich auf empirische Arbeiten, die im Zuge mehrerer Projekte entstanden sind. ${ }^{9}$ Für die Dokumentenanalyse wurden die Stellungnahmen der Ethikräte zu biomedizinischen Themen und Sitzungsprotokolle - soweit zugänglich - berücksichtigt. Außerdem wurden insgesamt 41 Leitfaden-Interviews mit Mitgliedern nationaler Ethikkommissionen in Deutschland, Österreich und der Schweiz durchgeführt. Die Interviewdauer betrug in der Regel anderthalb bis zwei Stunden. Die Interviews wurden vollständig transkribiert und in Anlehnung an die Strategie von Meuser und Nagel (2005) mithilfe der Software „Atlasti“" ausgewertet.

\section{„Nicht besonders professionalisiert“: Zur Relevanz ethischen Wissens}

Die konkreten bioethischen Streitfragen mögen die Mitglieder der nationalen Ethikräte regelmäßig entzweien, über eines besteht weitgehend Konsens: dass sich ethische Deliberation innerhalb der Kommissionen in der Regel auf einem common-sense-Niveau abspielen. Eine hohe Theorieladung der Debatte gilt überwiegend als unnötig, wenn das Ziel in der Verdichtung des Gruppendissenses zu einigen wenigen Positionen besteht. Schließlich ließen sich gremieninterne Koalitionen gerade auf Basis pragmatischer Diskussion realisieren - Koalitionen zwischen Kommissionsmitgliedern, die auf fundamentalethischer Ebene vielleicht

9 Dabei handelt es sich um das vom BMBF im Rahmen der Förderinitiative „Wissen für Entscheidungsprozesse“ finanzierte Projekt „Expertenwissen, Öffentlichkeit und politische Entscheidung“ (2004-2007), an dem Wolfgang Menz und Wilhelm Schumm vom Institut für Sozialforschung in Frankfurt am Main beteiligt waren, sowie das durch ein APART-Stipendium der Österreichischen Akademie der Wissenschaften geförderte Projekt „Soziologie bioethischer Expertise“ (2007-2009). 
weit entfernten Positionen angehören. Zu diesem Zweck erscheint es kontraproduktiv, immer gleich die Frage mit zu thematisieren, welche theoretischen Begründungstraditionen den jeweiligen Positionen entsprechen. In diesem Sinne sind Kant, Mill oder Rawls für ethische Deliberation weder unbedingt notwendig noch hilfreich. Gemeinsame Positionierungen lassen sich über weite Strecken eher auf „,mittlerer Ebene“ erarbeiten, wie es ein Mitglied des Nationalen Ethikrats formuliert.

„,Wir müssen jetzt, um zu einem Konsensus in bestimmten Positionen zu kommen, nicht gleich einen Konsensus haben auf der Meta-Ethik. Ja, also wir müssen jetzt nicht sagen, wir müssen jetzt erst zwischen John Stewart Mill und Immanuel Kant entscheiden, um sagen zu können, dass wir gegen den Missbrauch von Menschen sind. Ja, es gibt also auf der mittleren Ebene gibt es schon einen Konsensus hinsichtlich der Prinzipien. "

Tiefschürfende Ethikkontroversen sind dysfunktional, wenn man ergebnisorientiert diskutieren und pragmatische Einigungen innerhalb recht enger Zeithorizonte finden muss. Ethikexpertise hat in der Regel den Auftrag, einflussreiche Argumentationen zu strukturieren und politische Handlungsempfehlungen zu entwickeln. Fundamentalethische Argumentationsund Begründungsversuche, Theorievergleiche und Methodenkritik sind kein aussichtsreicher Weg für die Bewältigung dieser Aufgabe. Im Aushandlungsprozess setzt sich darum eine Pragmatisierung ethischer Deliberation durch. Das heißt, ethische Aspekte werden vor allem pragmatisch abgehandelt. Aus organisationssoziologischer Perspektive kann man diese Pragmatisierung als Resultat der Vermittlung wissenschaftlicher Exaktheitsansprüche und realpolitischer Anschlussfähigkeit innerhalb der Organisation Ethikrat lesen. Im Fall der Ethik stehen dabei wissenschaftliche und politische Ansprüche nicht komplementär, sondern konträr zueinander: Die Notwendigkeit interner Koalitionsfähigkeit und realpolitischer Orientierung steht Bemühungen ethischer Differenzierung entgegen. Im Fall naturwissenschaftlicher oder juristischer Expertise verhält sich dies - wie wir noch sehen werden - anders. Doch die Aushandlung von Wertfragen in Ethikräten erreicht nicht die Ebene, wo Ethik als disziplinäres Sonderwissen relevant werden könnte. In dem folgenden Zitat eines Mitglieds des Nationalen Ethikrats kommt dieser Effekt der Pragmatisierung von Ethikdebatten beispielhaft zum Ausdruck.

„Also sozusagen die Mediziner haben ihren Bereich, die Juristen haben eine sehr starke Position. Und die ganzen ethischen Fragen werden auf dem Niveau von reflektiertem Alltagsverständnis behandelt. Und da finde ich nicht, dass das besonders professionalisiert ist. “

Die Einschätzungen der Mitglieder lassen sich durch die Analyse der Berichte und Stellungnahmen nationaler Ethikräte erhärten. Ethische Aspekte werden immer schon mit Blick auf den konkreten Problembereich debattiert und erhalten eine stark anwendungsorientierte Note. Grundlagenethische Positionsbestimmungen haben in diesen Expertisen keinen Platz. Diese Pragmatisierung gilt im Prinzip für alle der untersuchten Bioethikkommissionen, wenn auch mit vereinzelten Ausnahmen. ${ }^{10}$ Für die professionelle Ethik bleibt da wenig Platz, wie ein Mitglied der österreichischen Kommission erklärt:

„Die Ethiker stellen ein theoretisches Instrumentarium zur Verfügung und es gibt ... sie stellen auch bestimmte Methoden zur Verfügung, die wir aber in der Kommission ja überhaupt nicht anwenden. Da wird eben herum diskutiert. Es ist ja nicht so, dass wir sozusa-

10 So findet sich in der zweiten Stellungnahme der Nationalen Ethikkommission der Schweiz zur Stammzellforschung (2002) eine Darstellung jener ethischen Leitmodelle (Kant, Utilitarismus, Verantwortungsethik), die der gremieninternen Orientierung in den Diskussionen dienten. Dies ist allerdings auch für Schweizer Verhältnisse eine Ausnahme, wenngleich die professionelle Ethik in dieser Kommission relativ stark vertreten ist. 
gen methodisch angeleitete Fallanalysen machen. Das machen wir ja dort gar nicht. Ich glaub', die professionelle Ethik, die ist irgendwie marginal. Aber das mag jetzt meine Einschätzung sein."

Die Marginalisierung der professionellen Ethik wird durch eine diskursiv stabilisierte und generalisierte Dissenserwartung in den Kommissionen forciert. Die Erwartung, dass sich in den abschließenden ethischen Positionierungen ein Dissens manifestieren wird, trägt zum pragmatischen Charakter der Diskussion bei. Zwar wird konzediert, dass es ethisch mehr oder weniger gut begründete Standpunkte gibt; andererseits aber existiert ein klares Bewusstsein darüber, dass es in ethischen Fragen stets mehrere legitime Standpunkte gibt. Nur eine starke Konsenserwartung könnte einen theoretischen Begründungszwang freisetzen, durch den ein ethisches Sonderwissen in besonderer Weise relevant würde. Doch aufgrund der Dissenserwartung muss man die Legitimität ohnehin geläufiger Werthaltungen nicht bis ins theoretische Detail argumentieren. Es reicht aus, die eigene Position durch sachliche Informiertheit und gegenseitige Kritik im Gremium robust zu machen. Diese Dissenserwartung schleift sich in der Kommissionsarbeit auch dort ein, wo sie anfangs vielleicht gar nicht bestand. So diskutierte die österreichische Kommission in ihrer Frühphase - sei es aufgrund des politisch verordneten Konsenszwangs, ${ }^{11}$ sei es aufgrund konsensueller Beschlüsse zuvor - das Stammzellen-Problem noch unter hoher Konsenserwartung. Die langwierigen, aber erfolglosen Versuche, eine gemeinsame Empfehlung zu formulieren, lassen sich als Lernprozess verstehen, in Folge dessen stärker auf Dissensorientierung umgeschaltet wurde. Dafür stehen etwa die wenig später einsetzenden Verhandlungen zur Präimplantationsdiagnostik (PID), in denen von Beginn an unter einer klaren Dissenserwartung operiert wurde.

Um es noch einmal zu betonen: Es ist nicht der Dissens an sich, der zur Relativierung ethischer Expertise beiträgt, sondern eine generalisierte Dissenserwartung, die innerhalb (vgl. van den Daele 2008), aber im Prinzip auch außerhalb der Expertenkommissionen vorherrscht: also der allgemeine Glaube an die Unüberwindlichkeit des Dissenses in ethischen Fragen sowie an das Fehlen einer übergeordneten, ordnungsstiftenden Instanz (Gott, Vernunft, Experten). Das heißt, im Fall von Wertedissens geht man gar nicht erst davon aus, durch den Rekurs auf widerstreitende Theorien und Paradigmen entscheiden zu können, wer Recht hat - den Dissens begreift man im Weiteren vielmehr als ein politisches Problem und vertraut auf entsprechende Aushandlungsprozesse, z.B. im Parlament.

Insgesamt wird deutlich und nachvollziehbar, dass in den Ethikkommissionen gegenüber ethischem Sonderwissen ein erheblicher Irrelevanzverdacht besteht. Die Ethiker werden in den Kommissionen denn auch weniger als Spezialisten wahrgenommen als die Vertreter anderer Disziplinen. Sie werden in den Deliberationsprozessen nicht als Ethikspezialisten adressiert; sie gelten den anderen Kommissionsmitgliedern eher als Menschen mit einem hohen Reflexionsniveau in ethischen Belangen - gewissermaßen als ethikbewusste Bürger.

„Bei den Ethikern ist es nicht so, dass man zum Beispiel sagt: Jetzt sag doch mal, was hat die deutsche Klassik dazu gesagt, oder was hat die antike Philosophie dazu gesagt, oder so. Das kommt eigentlich... also die diskutieren weniger eigentlich aus ihrer Wissenschaft heraus [...] Sondern mehr so allgemein, als dass sie jetzt als Spezialisten auftreten würden. Während die anderen Wissenschaften eigentlich schon als Spezialisten auftreten."

Auf Kommissionsebene tragen die Notwendigkeit zur Koalitionsbildung sowie die generalisierte Dissenserwartung zu einem Pragmatisch-Werden ethischer Deliberation bei, die letzt-

11 In $§ 7$ (2) der Einsetzungsverordnung heißt es, die Kommission habe ,bei der Beschlussfassung einen größtmöglichen Konsens anzustreben." Dass aus dem realpolitischen Selbstverständnis nationaler Ethikräte ein gewisser Zwang zum Konsens resultiert, liegt nahe. Als von der Politik verordnete Aufgabe ist dies eine Ausnahme. 
lich die Relevanz ethischen Sonderwissens in Frage stellt. Für die anderen Experten heißt das: Die Ethik wird nicht zur Superdisziplin, die die Reflexionsstandards bestimmt oder Professionalisierungszwänge freisetzt. Niemand muss nachts bei Kant und Rawls nachlesen, um am nächsten Tag in der Kommissionssitzung bestehen zu können. „Ethik“ beschränkt sich auf das Niveau der allgemeinen Ansprüche an Konsistenz und interne Logik der Argumente und Standpunkte. Im Übrigen werden auch von Seiten der beteiligten Ethiker keine Ansprüche auf eine Sonderstellung in den Gremien erhoben.

\section{„,We are all ethicists “? Grenzen zwischen Laien und Experten}

Ethische Problemstellungen werden nicht als reine Professionsdomäne oder aber als eine unverhandelbare Privatangelegenheit begriffen; kein Kommissionsmitglied versteht Ethik in dieser Weise. Vielmehr bringt die Ethik andere Disziplinen und Wissensformen als gleichwertige Gesprächspartner über Wertefragen ins Spiel. Eine von generalisierter Dissenserwartung getragene Wertedebatte schafft Anschlussstellen für die Beteiligung und Aufwertung von Ethiklaien. Mit anderen Worten: Ethisierung erzwingt Laienbeteiligung; sie lädt zum Mitreden ein, weil die Meinungen als Meinungen Geltung haben - und nicht durch ethisches Spezialwissen unterfüttert werden müssen (eher schon durch ein entsprechendes Sachstandswissen). Damit bringt Ethisierung eine tendenzielle Grenzauflösung zwischen Experten und Laien zum Ausdruck bzw. forciert diese. Im Lichte dieser Emanzipation der Laien kann man die Beratungen der Ethikräte durchaus als „Symmetrisierungsveranstaltung“ (Saake / Kunz 2006: 49) bezeichnen. Auf Seiten der Ethiker macht sich dies als Infragestellung ihrer Expertenschaft bemerkbar. Im Kommissionsalltag wird nicht recht klar, welche spezifische Expertise Ethiker exklusiv mobilisieren können, und manches Kommissionsmitglied fragt sich, was eigentlich deren wissenschaftliche Spezialisierung ist:

„Bei den Ethikern ist es, glaube ich, so, dass die einfach weniger spezialisiert sind, oder dass die... [...] Die Kerngruppe, die die Ethik vertritt... Bei denen weiß ich nicht genau, was eigentlich ihre wissenschaftliche Spezialisierung ist. "

Ganz offensichtlich können Ethikräte mit ihren Ethikern recht wenig anfangen, sofern es nicht um prozedurale Aufgaben geht. Als Moderatoren, die im Deliberationsprozess für Fairness sorgen, sowie als Redakteure der Stellungnahmen sind die Ethiker durchaus gefragt; sie gelten als nützlich, um die einzelnen Positionen innerhalb der Kommission ,in ein gutes wording zu verpacken“, wie es ein österreichisches Kommissionsmitglied formuliert, aber nicht als Ethikspezialisten. Schließlich geht es lediglich um argumentativ einigermaßen haltbare Positionierungen in konkreten Wertfragen und nicht um ethische Theoriebildung. Die Frage der ethischen Positionierung können Ethiker aber nicht monopolisieren: Hier beanspruchen vielmehr alle Mitglieder der Ethikräte über entsprechende Kompetenzen zu verfügen. Und nicht nur die Mitglieder von Ethikräten - gerade in bioethischen Fragen wird der Ethikexpertise von informierten Laien, die von Partizipationsprofis etwa im Rahmen von Bürgerkonferenzen mobilisiert werden (Abels / Bora 2004), ein erheblicher Orientierungswert zugeschrieben. Darum lautet auch die Standard-Suggestivfrage in Talkshows und Parlamenten, sobald es um die Einrichtung von nationalen Ethikräten geht: Sind wir in Sachen Ethik denn nicht alle Experten ${ }^{12}$

12 Nicht einmal die Vorsitzenden von Ethikkommissionen können sich einer positiven Antwort entziehen. Um ein illustres Beispiel zu zitieren: Die Baroness Kennedy, ehemalige Vorsitzende der Human Genetics Commission, sagt im House of Lords zur Frage, ob man in England eine nationale Bioethikkommission brauche: „I am unhappy about subcontracting ethics to a group of ethicists. [...] we are all ethicists." (House of Lords, 28.1.2008, Human Fertilisation and Embryology Bill, Vol. 698, cols 489 f.). 
Diese Grenzauflösung zwischen Laien und Experten wird nicht zuletzt durch die spezifische Logik ethischer Erkenntnisplausibilisierung stabilisiert. Erkenntnisplausibilisierung funktioniert in der Ethik als Prozess, der in der Interaktion zwischen Experten und Laien in Echtzeit abläuft und nicht - wie in den Naturwissenschaften - durch den Verweis auf „Fakten" oder stabilisierte Funktionszusammenhänge abgekürzt werden kann. Ethiker können ihr Wissen nicht als „black box“ vorführen. Demgegenüber sind die Naturwissenschaften abstrakt, d.h. sie unterhalten von der Lebenswelt abgeschirmte Laborwelten mit entsprechend künstlichen Objekten. Die einzelnen Schritte ihrer Wahrheitsproduktion sind von außen nicht unmittelbar nachvollziehbar - man muss vertrauen (und tut es in der Regel leichten Herzens, weil das naturwissenschaftliche Wissen im Rahmen von Ethikexpertise meist nur als kanonisiertes Wissen relevant wird). Ethische Argumente muss man nicht glauben, sie müssen überzeugen. Die Plausibilität der Ethik ergibt sich über die In vivo-Darstellung des ausgeführten Arguments. Die Ethiklaien können daher nicht auf Distanz gehalten, sie müssen einbezogen werden.

So sehen sich die Ethiklaien in den Ethikräten in die Lage versetzt, die Gültigkeit ethischer Argumentationen selbständig zu beurteilen und herauszufordern. Das heißt in der Praxis natürlich nicht, dass die Authentizität eines Kant-Zitats in Frage gestellt wird. Doch es wird geprüft, ob die damit korrespondierende Argumentation plausibel ist. In diesem Sinne werden ethische Argumentationen einer Plausibilitätskontrolle durch die Laien geöffnet. Dies ist es, was einleitend unter dem Aspekt der Robustheit subsumiert wurde, auch wenn an dieser Stelle die Schwierigkeiten einer Typisierung von Geltungskriterien offensichtlich werden. Denn vielleicht ist Robustheit hier weniger als eigenständige Geltungsdimension, sondern eher als Variante einer Wahrheitsprüfung (durch Laien) zu verstehen. In jedem Fall lässt sich festhalten, dass - mit Ausnahme der Theologie ${ }^{13}$ - kaum eine andere Disziplin innerhalb der Ethikräte sich derartigen Geltungsvorbehalten ausgesetzt sieht wie ausgerechnet die Ethik.

\section{„Nationaler Rechtsrat"? Die Dominanz der Jurisprudenz}

Die relative Marginalisierung fachethischen Wissens wird in besonderer Weise durch den Vergleich mit juristischer Expertise deutlich sowie durch deren Dominanz forciert. Im Folgenden wird gezeigt, dass die den Juristen zugeschriebene Bedeutung eine Tendenz zur Verrechtlichung eines an sich durch Ethisierung geprägten Diskurses begründet. Verrechtlichung meint in diesem Zusammenhang ein teilweises Reframing ethischer Deliberation nach Maßgabe des juristischen Relevanzsystems. ${ }^{14}$

Juristische Expertise erhält innerhalb der nationalen Ethikräte eine starke Stellung, weil sich die Mitglieder in den Aushandlungsprozessen auf etwas festgelegt sehen, was man ethische Realpolitik nennen könnte. Im Rahmen dieser ethischen Realpolitik wird juristischer Expertise eine hohe Praxis- und Entscheidungsrelevanz zugeschrieben. Die relevanten Rechtsnormen, also Gesetze, Rechtsverordnungen und natürlich - gerade in Deutschland in Lebensfragen an erster Stelle - die Verfassung werden zum archimedischen Punkt der kommissions-

13 Die Theologen sehen sich gezwungen, ihre theologischen Standpunkte säkular-ethisch zu reformulieren. Ein Ethikrat sei schließlich, so der Tenor unter den Mitgliedern, nicht ein Konzil, sondern ein Ethics Council. Daher kommen weder in den Diskussionen noch in den Stellungnahmen Gott und die Offenbarung vor. Dies wird von anderen Kommissionsmitgliedern, denen selbst nichts an religiösen Begründungen liegt, zuweilen als Korrumpierung der Theologen bewertet: „Aber es erschreckt mich trotz allem, sage ich Ihnen schon, wenn ich auch von keinem Vertreter der Kirchen ein einziges Mal das Wort Gott gehört habe. Existiert nicht! Verstehen Sie?“.

14 Vgl. mit ähnlicher Stoßrichtung Bora (2006), der am Beispiel der grünen Gentechnik zeigt, dass die qua institutionalisierter Bürgerbeteiligung erhoffte Politisierung durch den rechtlichen Rahmen neutralisiert wird. 
internen Aushandlungen. ${ }^{15}$ Juristisches Wissen verspricht einer unübersichtlichen und prinzipiell unendlichen Wertedebatte ein festes Fundament zu verleihen. Andererseits wird dieser Bezug auf geltendes Recht natürlich wichtig, wenn sich Expertise zwecks politischer Relevanz in die Rechtsordnung einfügen muss - auch oder gerade wenn man Reformen anstrebt. In Form der Aufklärung über kodifizierte, rechtsverbindliche Normen und die maßgebliche Rechtsprechung bestimmen die Juristen immer auch, was als Empfehlung der Kommission an geltende Normvorstellungen und bestehendes Recht anschlussfähig und damit auch politikrelevant ist. Sie definieren sozusagen den Spielraum des „ethisch Möglichen“ und damit den Bereich relevanter Reflexion. Sie sind, wie ein Ethikrats-Mitglied aus dem Bereich der Rechtswissenschaften sagt, die „Grenzer“.

„,Wir sind, wenn Sie so wollen, die Grenzer. Also wir sagen zu beiden Seiten hin, das kannst du so meinen, aber du kannst es nicht durchsetzen. [...] Wir sind diejenigen, die verhindern, dass ein Thema von einer ethischen oder theologischen Seite so in Anspruch genommen wird, dass dies für die anderen nicht mehr akzeptabel ist."

Die Juristen verkörpern nach dieser Lesart das „Realitätsprinzip“, weil das Recht gewissermaßen den ethischen Minimalkonsens repräsentiert. Die Juristen erscheinen als diejenigen, die im Sinne der freiheitlichen Grundordnung das Gemeinwesen gegen potenzielle „Fundamentalisten“ aus Ethik und Theologie schützen. In Summe jedenfalls können die Juristen aufgrund der ihrem Sonderwissen zugeschriebenen Praxisrelevanz besondere Expertiseansprüche durchsetzen. Die von vielen Kommissionsmitgliedern als „stark“ charakterisierte Stellung der Juristen ist jedoch nicht durch eine besondere Eindeutigkeit oder Verlässlichkeit ihres Wissens begründet. Sie scheint vielmehr in den allgemeinen Erwartungen zu wurzeln, die sich an dieses Wissen richten, nämlich der „weichen“ Ethikmaterie einen klaren Rahmen zu geben. ${ }^{16}$

Weil das Wissen der Juristen eben als Sonderwissen für die Kommissionen relevant wird, wird die Grenze zwischen Experten und Laien stabil gehalten. Diskussionen über die Rechtslage im In- und Ausland als auch rechtshermeneutische Streitigkeiten bezeichnen Verhandlungsfelder, auf denen die Juristen unter sich bleiben. Aufgrund des hohen Niveaus der juristischen Debatte, die nicht zuletzt mit der besonderen Politikrelevanz des Rechts zu tun hat, ist disziplinäres Spezialwissen zur Teilhabe an diesen Diskussionen notwendig. Das hat zur Folge, dass die Expertise der Juristen von außen nicht leicht bestritten werden kann. Kritik an der Jurisprudenz äußert sich in den Ethikräten dementsprechend eher als Meta-Kritik: Kritisiert wird etwa die Monopolisierung ethischer Deliberation durch die Juristen; die ethische Debatte um das normativ Wünschenswerte verschiebe sich, so der Tenor, oft hin zu einer Diskussion um das gegenwärtig Erlaubte. Wie die folgende Passage aus einem Interview mit einem Fachethiker zeigt, wehren sich gerade die Ethiker gegen das Deutungsmonopol der Juristen - mit eher geringer Aussicht auf Erfolg, sofern unsere Analyse schlüssig ist.

15 Der Stellenwert juristischer Argumentationen ist in Deutschland und Österreich ähnlich groß, auch wenn die Bedeutung der Verfassung deutlich differiert. So gibt es in Österreich weder ein vergleichbares Verfassungsethos noch eine verfassungsrechtliche Garantie der Menschenwürde; diese ergibt sich allenfalls indirekt über den Bezug auf die Europäische Menschenrechtskonvention, die sich freilich kaum auf Embryonen beziehen lässt, vgl. Kopetzki (2003: 63 f.).

16 Die Dominanz des Rechts dürfte in jenen biopolitischen Bereichen, die nicht so eindeutig ethisch gerahmt sind wie die Fragen der Embryonenforschung, noch deutlicher sein. Zum Beispiel Biobanken. Der bei diesem Thema weitgehende Konsens innerhalb der Kommissionen scheint denn auch nicht zuletzt auf der Homogenisierungswirkung des rechtlichen Rahmens zu beruhen. So erklären Kommissionsmitglieder, sie hätten trotz Bauchschmerzen der Gemeinschaftsposition zugestimmt, weil der Aufwand für die Fundierung einer haltbaren Rechtsposition, die den eigenen Standpunkt besser abbilden würde, zu hoch sei. 
„Die Juristen aber neigen dazu, die Fragen als rein juristisch anzusehen und sie damit dann auch schon für erledigt zu halten. Und neigen manchmal dazu, auch ein zusätzliches moralisches Argument als überflüssig anzusehen, weil man es für den Gesetzgebungsprozess doch gar nicht braucht. Und da muss man sich dann behaupten. [...] Da bin ich im ständigen Kampf auch für Einsichten, die ... der Ethikrat, das ist ja kein deutscher nationaler Rechtsrat, sondern es ist ja ein Ethikrat, dass diese Dimension nicht verloren geht. “

Die Juristen verfügen über ein Sonderwissen, das in seiner Relevanz und Geltung durch die Logiken der gremieninternen Interaktionsprozesse nicht relativiert wird. Und sofern das Wissen als Sonderwissen relevant bleibt, kann es von disziplinären Laien nicht wirkungsvoll in Frage gestellt werden. Genau das macht den Expertenstatus der Juristen und starke Disziplinen wie die Rechtswissenschaften aus: Auseinandersetzungen um Geltungsansprüche des Wissens werden fast ausschließlich als interner Streit vorgeführt. Im Kontext dieser Geltungsverhältnisse stabilisiert sich das Deutungsmonopol der Juristen: Ihre Wirklichkeitskonstruktionen werden handlungs- und orientierungsleitend für andere. Die Relevanz scheint somit das für Geltungsfragen entscheidende Kriterium in interdisziplinären Gremien zu sein. Wahrheit und Robustheit des Wissens können durch einen Irrelevanzverdacht unterlaufen werden. Die Anerkennung der Relevanz disziplinären Sonderwissens aber stabilisiert die Differenz zwischen Laien und Experten.

Dazu mag im Übrigen auch die Konsenserwartung beitragen, die gegenüber dem Recht besteht. In Bezug auf die Ethik war von einer generalisierten Dissenserwartung die Rede. In Rechtsfragen besteht dagegen die Erwartung, dass mit Hilfe fachspezifischer methodischer Standards ein wenigstens temporärer Konsens hergestellt werden kann. Diese Erwartung mag nicht zuletzt durch praktische Anschauung geprägt sein. Tatsächlich wird in der Praxis Dissens, wenn er in Verbindung mit dem Grundgesetz oder Landesverfassungen steht, mit Hilfe des Verfassungsgerichts zumindest temporär überwunden - also mit den Mitteln des Rechts. In ethischen Fragen scheint ein vergleichbarer Schließungsmodus der Konflikte, z.B. durch nationale Ethikräte, nicht möglich. Ethikräte haben weder die formale Autorität noch die Glaubwürdigkeit, Ethikfragen verbindlich zu regeln; sie glauben selbst nicht an die Überlegenheit ihrer Empfehlungen. In der Praxis verdoppeln sie lediglich den real existierenden Dissens. Die Schließung des Wertkonflikts wird damit zur politischen Aufgabe. Kurz: Es gibt im Fall des Rechts Konsenserwartungen, die dauerhaften Dissens illegitim machen und die Überwindung des Dissenses nur mit Hilfe juristischer Methoden legitim erscheinen lasen. In jedem Fall existieren in Ethikräten offenbar disziplinspezifisch variierende Dissenserwartungen. Naturwissenschaftliche oder rechtliche Sachstände werden in den Stellungnahmen der Ethikräte - anders als die Einlassungen zur Ethik - nicht schon im Plural abgefasst. Für interdisziplinäre Gremien müsste das heißen: Der Geltungs- und Expertisestatus korreliert positiv mit den Konsenszuschreibungen an die Disziplin.

Die These von der Macht der Juristen in den Kommissionen lässt sich noch zuspitzen. Man könnte etwa von der Marginalisierung der Ethik durch das Recht sprechen. Das heißt, die Ethik bleibt zwar als Rahmen in einem abstrakten Sinne relevant, doch die konkreten 
Debatten werden in den Bahnen des juristischen Relevanzsystems geführt. ${ }^{17}$ Dafür sind zum einen die kategorialen Konvergenzen zwischen Recht und Ethik maßgeblich. In den Aushandlungsprozessen der Kommissionen wird deutlich, dass keine scharfen Grenzen zwischen Ethik und Recht existieren. Es ist möglich, ethische Debatten in juristischen Begriffen zu reformulieren (Menschenwürde, Forschungsfreiheit, Recht auf Leben usw.); und es gibt juristische Pendants zu ethischen Konzepten, wie etwa das Recht auf körperliche Unversehrtheit, das geeignet ist, all jene Tatbestände aus rechtlicher Perspektive zu thematisieren, die dem ethischen Prinzip des Nicht-Schadens entsprechen. Dem ethischen Diskurs werden also juristische Begrifflichkeiten und Denkmuster nicht einfach übergestülpt. Vielmehr werden ethische Argumente aus rechtswissenschaftlicher Perspektive rezipiert, angeeignet, reformuliert und damit dem juristischen Relevanzrahmen subsumiert.

Zum anderen trägt gerade der Dissens innerhalb der anwesenden Juristen zur Marginalisierung der Ethik bei. Dissens ergibt sich aus verschiedenen Gründen, aufgrund von Interpretationsspielräumen, aber auch aufgrund unterschiedlicher Spezialisierungen und Traditionen. In den Ethikräten sitzen Verfassungsrechtler, Medizinrechtler und Datenschützer mit jeweils ganz unterschiedlichen normativen Bezugspunkten und Rechtsauslegungen am Tisch. Entsprechend heterogen sind die Einschätzungen über die Bedeutung des Rechts im konkreten Fall.

„Dann sagt zum Beispiel ein Medizinrechtler, dass das eben sozusagen aus fachjuristischer Sicht nicht geht, und der Verfassungsjurist sagt: Das muss gehen, ja, weil zum Beispiel die Freiheit der Forschung in Artikel 5 des Grundgesetzes festgehalten ist und nicht in Artikel 91. Und da kann man nicht jetzt sagen, wir betrachten die Hierarchisierung nicht. - So bringen dann die Leute... oder die Praktiker sagen: Da gibt es aber schon 70 Gerichtsentscheide, die in eine andere Richtung gehen, und die können wir nicht einfach ignorieren."

Derlei Problemstellungen machen deutlich, dass die juristischen Auseinandersetzungen auf der Höhe der rechtswissenschaftlichen Debatte geführt werden. Von einer Pragmatisierung der rechtlichen Debatte kann nicht die Rede sein, im Gegenteil. Dabei verhindert der Dissens unter den Juristen eine frühzeitige Schließung der Debatte unter Verweis auf gültige Rechtsnormen und ermöglicht damit im Weiteren eine Übersetzung ethischer Fragen in juristisch zu verhandelnde Problemstellungen. Erst der Dissens macht es möglich, ethische Fragen als juristische Rechtsauslegungsdebatte führen zu können.

\section{Resümee}

Zusammenfassend lässt sich ein paradoxer Zusammenhang konstatieren: Einerseits beobachten wir - gewissermaßen auf der Makro-Ebene - ein Ausgreifen der Ethik, das unter anderem die Forschungs- und Technikregulierung als Gegenstand ethischer Reflexion kennzeichnet (Ethisierung). Andererseits macht die hier präsentierte Mikro-Analyse deutlich, dass die Organisation der ethischen Reflexion in Form interdisziplinärer Expertengremien zu einer relativen Marginalisierung ethischen Sonderwissens führt. Das heißt, die Ethisierung führt gerade nicht dazu, dass die Ethik ein besonderes Deutungsmonopol für die Reflexion ethischer

17 Alle Stellungnahmen der Ethikräte sind von einer starken juristischen Handschrift geprägt. Die Ausführungen zur rechtlichen Situation sind stets ausführlich und nehmen vielfach bereits ethische Aspekte vorweg. In vielen Berichten (nicht allen!) gibt es einen eigenständigen Ethikteil. Doch oftmals ist der Bezug auf Ethik nur um den Preis von Redundanzen möglich. So wird in der Empfehlung des Nationalen Ethikrats zum Stammzell-Import zum Beispiel die Widersinnigkeit eines vollen Lebensschutzes für Embryos zuerst juristisch und dann noch einmal ethisch begründet, obwohl die Argumente - der Embryo sei, siehe Abtreibung oder Nidationshemmer, in vitro besser geschützt als in vivo - identisch sind (Nationaler Ethikrat 2002: 19ff). 
Fragen durchsetzen könnte. Denn im Ergebnis sind die Profi-Ethiker in den Ethikräten von diskursiv gleichwertigen Ethiklaien umgeben, die auf Augenhöhe mitreden können, weil ethische Deliberation sich - aus verschiedenen Gründen - auf einem common-sense-Niveau abspielt. Aus dieser Perspektive erscheint der Ruf nach einer stärkeren Besetzung der Ethikräte mit Ethikern (der z.T. auch aus den Reihen der Ethikräte selbst kommt) als so verständlich wie vergeblich. Kommissionsethik funktioniert nicht qua überlegener oder auch nur elaborierter ethischer Moralbegründung, sondern vermittels der Pragmatisierung ethischer Deliberation. Aus organisationssoziologischer Perspektive liest sich dies als Resultat der Vermittlung wissenschaftlicher und politischer Ansprüche, die den Ethikräten als „boundary organizations" aufgegeben sind.

Aus mikropolitischer Perspektive lässt sich diese Kommissionsethik als Interaktionsgeflecht verstehen, in dem sich Machtbeziehungen zwischen den einzelnen Experten und Disziplinen konstituieren. Diese gründen ganz wesentlich auf der Geltung des Wissens, die in den Dimensionen von Wahrheit, Robustheit und Relevanz aufgeschlüsselt wurde. Ethikexpertise ist nicht gleichbedeutend mit einem zwanglosen, herrschaftsfreien Diskurs unter gleichberechtigten Fachexperten - und kann es nicht sein. Es wurde vielmehr deutlich, dass Mikropolitik zwangsläufige Folge einer Expertise ist, die als Aushandlungsprozess zwischen Vertretern verschiedener Disziplinen und Weltanschauungen organisiert ist. In diesem Prozess greifen Koalitionsbildungen, Marginalisierungen und Strategien, die aus bestimmten Erwartungshaltungen herrühren. Mit letzterem Aspekt sind Strukturbedingungen des Handelns in den Blick geraten, die es verbieten, Machtbeziehungen allein als Ergebnis strategisch-intentionaler Konkurrenzen zwischen Fachexperten zu verstehen. Diese erscheinen vielmehr ebenso als Effekt von Erwartungsstrukturen, die eine erhebliche Bedeutung für die Einschränkung der Kontingenz haben.

Abschließend wäre zu überlegen, auf welche Weise diese empirische Analyse zu einer noch zu begründenden Soziologie der Ethik beiträgt. In diesem Beitrag wurden die epistemischen und institutionellen Bedingungen analysiert, unter denen Ethikexpertise entsteht und Geltung erlangt. Eine Soziologie der Ethik muss darüber hinaus greifen. Sie könnte in zeitdiagnostischer Absicht das Verblassen der Risikogesellschaft mit der Heraufkunft einer ,Ethogesellschaft" in Verbindung bringen. Damit wäre ein Projekt definiert, das die Soziologie der Ethik in mehreren Dimensionen in Angriff nehmen könnte: Sie hätte sich mit der Frage zu befassen, auf welche Weise und in welcher Form die Ethik (als akademischer Diskurs, politikrelevante Expertise, Governance-Semantik...) sich in sozialen Systemen etabliert und Geltung erlangt. Sie hätte insbesondere das konjunkturelle Auf und Ab der Ethik im Verhältnis zu der sich wandelnden Ordnung der Gesellschaft zu analysieren und auf diese Weise den Funktionswandel der Ethik zu erklären. Vor allem aber hätte sie zu analysieren, welche Folgen die aktuell zu beobachtende Ethisierung für bestehende institutionelle Architekturen, Konfliktkonstellationen, Politik- und Legitimationsmuster hat. Erste Ansätze dazu finden sich in jenen Analysen, die die Subjektivierung politischer Regulation als Ethopolitik (N. Rose) fassen. In einem solchen Arbeitsprogramm hat dann natürlich auch die Soziologie der Ethikexpertise ihren Platz: Sie hätte die Hochkonjunktur der Ethikkommissionen im Hinblick auf veränderte Legitimationsansprüche und sich wandelnde Governance-Modelle in den verschiedenen Bereichen der Gesellschaft zu analysieren.

\section{Literatur}

Abels, Gabriele / Alfons Bora (2004): Demokratische Technikbewertung, Bielefeld.

Bogner, Alexander / Wolfgang Menz / Wilhelm Schumm (2008): Ethikexpertise in Wertkonflikten. Zur Produktion und politischen Verwendung von Kommissionsethik in Deutschland und Österreich, in: 
Renate Mayntz / Friedhelm Neidhardt / Peter Weingart / Ulrich Wengenroth (Hrsg.), Wissensproduktion und Wissenstransfer. Wissen im Spannungsfeld von Wissenschaft, Politik und Öffentlichkeit, Bielefeld, S. 243-268.

Bora, Alfons (2006): Im Schatten von Normen und Fakten - Die Kolonisierung der Politik durch technowissenschaftliche Normativität, in: Zeitschrift für Rechtssoziologie 27, S. 31-50.

Bora, Alfons (2009): Wissenschaftliche Politikberatung und die disziplinären Grundlagen der Wissenschaft, in: Alexander Bogner / Karen Kastenhofer / Helge Torgersen (Hrsg.), Inter- und Transdisziplinarität im Wandel? Neue Perspektiven auf problemorientierte Forschung und Politikberatung, BadenBaden (im Erscheinen).

Bosetzky, Horst (1977): Machiavellismus, Machtkumulation und Mikropolitik, in: Zeitschrift für Organisation 46, S. 121-125.

Braun, Kathrin / Svea Luise Herrmann / Sabine Könninger / Alfred Moore (2008): Die Sprache der Ethik und die Politik des richtigen Sprechens. Ethikregime in Deutschland, Frankreich und Großbritannien, in: Renate Mayntz / Friedhelm Neidhardt / Peter Weingart / Ulrich Wengenroth (Hrsg.), Wissensproduktion und Wissenstransfer. Wissen im Spannungsfeld von Wissenschaft, Politik und Öffentlichkeit, Bielefeld, S. 221-242.

Clarke, Wiliam C. / Giandomenico Majone (1985): The Critical Appraisal of Scientific Inquiries with Policy Implications, in: Science, Technology \& Human Values 10, S. 6-19.

Collins, Harry / Robert Evans (2007): Rethinking Expertise. Chicago - London.

Crozier, Michel / Erhard Friedberg (1993): Die Zwänge kollektiven Handelns - Über Macht und Organisation (Neuausgabe). Frankfurt / Main.

Dahinden, Urs (2006): Framing. Eine integrative Theorie der Massenkommunikation, Konstanz.

Elšik, Wolfgang (1997): Zwischen Handlung und Struktur: Ansätze zur Politik in Organisationen, in: Kurswechsel 11, S. 9-22.

Entman, Robert M. (1993): Framing: Toward Clarification of a Fractured Paradigm, in: Journal of Communication 43, S. 51-58.

Fleck, Ludwik (1994): Entstehung einer wissenschaftlichen Tatsache - Einführung in die Lehre vom Denkstil und Denkkollektiv, Frankfurt / Main.

Foucault, Michel (1983): Der Wille zum Wissen. Sexualität und Wahrheit 1, Frankfurt / Main.

Foucault, Michel (1994): Das Subjekt und die Macht, in: Hubert L. Dreyfus / Paul Rabinow (Hrsg.), Michel Foucault. Jenseits von Strukturalismus und Hermeneutik. Weinheim, S. 243-261.

Friedberg, Erhard (1992): Zur Politologie von Organisationen, in: Willi Küpper / Günther Ortmann (Hrsg.), Mikropolitik. Rationalität, Macht und Spiele in Organisationen, 2. Auflage, Wiesbaden, S. 39-52

Fuchs, Michael (2005): Nationale Ethikräte. Hintergründe, Funktionen und Arbeitsweisen im Vergleich, Berlin: Nationaler Ethikrat.

Galloux, Jean-Christophe / Arne Thing Mortensen / Suzanne De Cheveigné / Agnes Allandsdottir / Aigli Chatjouli / George Sakellaris (2002): The institutions of bioethics, in: Martin W. Bauer / George Gaskell (Hrsg.), Biotechnology. The Making of a Global Controversy, Cambridge, S. 129-148.

Gill, Bernhard (2003): Streitfall Natur. Weltbilder in Technik- und Umweltkonflikten, Wiesbaden.

Guston, David H. (2001): Boundary Organizations in Environmental Policy and Science: An Introduction, in: Science, Technology, and Human Values 26, S. 399-408.

Jasanoff, Sheila (1985): Peer Review in the Regulatory Process, in: Science, Technology \& Human Values 10, S. 20-32.

Jung, Arlena (2009): Identität und Differenz. Sinnprobleme der differenzlogischen Systemtheorie, Bielefeld. 
Kneer, Georg (2008): Institution / Organisation: Über die Paradoxie des Organisierens, in: Stephan Moebius / Andreas Reckwitz (Hrsg.), Poststrukturalistische Sozialwissenschaften. Frankfurt / Main, S. 124-140.

Knights, David (1997): Organization Theory in the Age of Deconstruction: Dualism, Gender and Postmodernism Revisited, in: Organization Studies 18, S. 1-19.

Knorr-Cetina, Karin (1984): Die Fabrikation von Erkenntnis - Zur Anthropologie der Naturwissenschaften. Frankfurt / Main.

Kopetzki, Christian (2003): Rechtliche Aspekte des Embryonenschutzes, in: Ulrich Körtner / Christian Kopetzki (Hrsg.), Embryonenschutz - Hemmschuh für die Biomedizin?, Wien, S. 49-72.

Küpper, Willi / Günther Ortmann (1986): Mikropolitik in Organisationen, in: Die Betriebswirtschaft 46, S. 590-602.

Lazega, Emanuel (1992): Micropolitics of Knowledge. Communication and Indirect Control in Workgrouops, New York.

Lentsch, Justus (2008): Qualitätssicherung in der wissenschaftlichen Politikberatung, in: Stephan Bröchler / Rainer Schützeichel (Hrsg.), Politikberatung. Stuttgart, S. 194-216.

Lindsey, Nicola / Mercy W. Kamara / Erling Jelsoe / Arne Thing Mortensen (2001): Changing Frames: the Emerging of Ethics in European Policy on Biotechnology, in: Politeia 17, S. 80-93.

Luhmann, Niklas (1997): Ethik in internationalen Beziehungen, in: Wilhelm Lütterfelds / Thomas Mohrs (Hrsg.), Eine Welt - eine Moral? Eine kontroverse Debatte. Darmstadt, S. 156-166.

Maasen, Sabine (2002): Die gesellschaftliche Disziplinierung bio- und gen-ethischer Fragen durch die politische Institutionalisierung von „Diskurs“, Eine Expertise für das Bundesministerium für Bildung und Forschung, abgerufen am 16.5.2009 unter http://www.sciencepolicystudies.de/dok/expertisemaasen.pdf.

Maasen, Sabine / Mario Kaiser (2007): Vertrauen ist gut. Verantwortung ist besser - Die Herstellung von Verantwortlichkeit in der partizipativen Technikfolgenabschätzung, in: Rouven Porz / Christoph Rehmann-Sutter / Jakie Leach Scully / Markus Zimmermann-Acklin (Hrsg.), Gekauftes Gewissen? Zur Rolle der Bioethik in Institutionen, Paderborn, S. 71-87.

Meuser, Michael / Nagel, Ulrike (2005): ExpertInneninterviews - vielfach erprobt, wenig bedacht. Ein Beitrag zur qualitativen Methodendiskussion, in: Alexander Bogner / Beate Littig / Wolfgang Menz (Hrsg.), Das Experteninterview - Theorie, Methode, Anwendung, 2. Aufl., Wiesbaden, S. 71-93.

Mittelstraß, Jürgen (1987): Die Stunde der Interdisziplinarität?, in: Jürgen Kocka (Hrsg.), Interdisziplinarität. Praxis - Herausforderung - Ideologie, Frankfurt / Main, S. 152-158.

Moorstedt, Tobias (2007): Ethisches Verbraucherverhalten, in: Heinrich Geiselberger (Hrsg.), Und jetzt? Politik, Protest und Propaganda, Frankfurt / Main, S. 281-293.

Mouffe, Chantal (2007): Über das Politische. Wider die kosmopolitische Illusion. Frankfurt / Main.

Nationale Ethikkommission im Bereich Humanmedizin (2002): Zur Forschung an embryonalen Stammzellen. Stellungnahme, Bern.

Nationaler Ethikrat (2002): Zum Import menschlicher embryonaler Stammzellen. Stellungnahme, Berlin.

Neuberger, Oswald (2006): Mikropolitik und Moral in Organisationen, 2. Auflage, Stuttgart.

Nowotny, Helga / Peter Scott / Michael Gibbons (2001): Re-Thinking Science - Knowledge and the Public in an Age of Uncertainty, Cambridge.

Nullmeier, Frank / Tanja Pritzlaff / Achim Wiesner (2003): Mikro-Policy-Analyse. Ethnographische Politikforschung am Beispiel der Hochschulpolitik, Frankfurt - New York.

Ortmann, Günther (1992): Handlung, System, Mikropolitik, in: Willi Küpper / Günther Ortmann (Hrsg.), Mikropolitik. Rationalität, Macht und Spiele in Organisationen, 2. Aufl., Wiesbaden, S. 217-225.

Rose, Nikolas (2001): The Politics of Life Itself, in: Theory, Culture \& Society 18, S. 1-30. 
Rubin, Beatrix P. (2008): Therapeutic Promise in the Discourse of Human Embryonic Stem Cell Research, in: Science as Culture 17, S. 13-27.

Saake, Irmhild / Dominik Kunz (2006): Von Kommunikation über Ethik zu „ethischer Sensibilisierung": Symmetrisierungsprozesse in diskursiven Verfahren, in: Zeitschrift für Soziologie 35, S. 41-56.

Schön, Donald A. / Martin Rein (1995): Frame Reflection: Toward the Resolution of Intractable Policy Controversies, New York.

Simmel, Georg (1958): Der Streit, in: Ders., Soziologie. Untersuchungen über die Formen der Vergesellschaftung, Berlin, S. 186-255.

Stehr, Nico (2006): Die Moralisierung der Märkte. Eine Gesellschaftstheorie, Frankfurt / Main.

Sutter, Barbara (2005): Von Laien und guten Bürgern: Partizipation als politische Technologie, in: Alexander Bogner / Helge Torgersen (Hrsg.), Wozu Experten? Ambivalenzen der Beziehung von Wissenschaft und Politik, Wiesbaden, S. 220-240.

Türk, Klaus (1993): Politische Ökonomie der Organisation, in: Alfred Kieser (Hrsg.), Organisationstheorien, Stuttgart, S. 297-332.

van den Daele, Wolfgang (2001): Von moralischer Kommunikation zur Kommunikation über Moral Reflexive Distanz in diskursiven Verfahren, in: Zeitschrift für Soziologie 30, S. 4-22.

van den Daele, Wolfgang (2008): Über den Umgang mit unlösbaren moralischen Konflikten im Nationalen Ethikrat, in: Dieter Gosewinkel / Gunnar Folke Schuppert (Hrsg.), Politische Kultur im Wandel von Staatlichkeit, Berlin, S. 357-384.

van den Daele, Wolfgang / Alfred Pühler / Herbert Sukopp (1996): Grüne Gentechnik im Widerstreit. Modell einer partizipativen Technikfolgenabschätzung zum Einsatz transgener herbizidresistenter Pflanzen, Weinheim.

von Glasersfeld, Ernst (1997): Wege des Wissens. Konstruktivistische Erkundungen durch unser Denken, Heidelberg.

Weingart, Peter / Martin Carrier / Wolfgang Krohn (2007): Nachrichten aus der Wissensgesellschaft. Analysen zur Veränderung der Wissenschaft, Weilerswist.

Weißbach, Barbara (2001): Expertenwelten und Managementpraxis: Zur Mikropolitik des Wissens, in: Arbeit 10, S. 167-177.

Dr. Alexander Bogner

Institut für Technikfolgen-Abschätzung (ITA) der Österreichischen Akademie der Wissenschaften

Strohgasse 45

1030 Wien

abogner@oeaw.ac.at 